\title{
A note on Gaussian correlation inequalities for nonsymmetric sets
}

\author{
Adrian P. C. $\operatorname{Lim}^{a}$, Dejun Luo ${ }^{a, b *}$ \\ ${ }^{a}$ UR Mathématiques, Université de Luxembourg, 6, rue Richard Coudenhove-Kalergi, L-1359 Luxembourg \\ ${ }^{b}$ Key Lab of Random Complex Structures and Data Science, Academy of Mathematics and \\ Systems Science, Chinese Academy of Sciences, Beijing 100190, China
}

\begin{abstract}
We consider the Gaussian correlation inequality for nonsymmetric convex sets. More precisely, if $A \subset \mathbb{R}^{d}$ is convex and the origin $0 \in A$, then for any ball $B$ centered at the origin, it holds $\gamma_{d}(A \cap B) \geq \gamma_{d}(A) \gamma_{d}(B)$, where $\gamma_{d}$ is the standard Gaussian measure on $\mathbb{R}^{d}$. This generalizes Proposition 1 in [Arch. Rational Mech. Anal. 161 (2002), 257-269].
\end{abstract}

Keywords: Correlation inequality, Gaussian measure, convexity, log-concavity, optimal transport

MSC 2000: 60E15, 26A51

\section{Introduction}

Let $A, B \subset \mathbb{R}^{d}$ be symmetric convex subsets. The Gaussian correlation inequality claims that

$$
\gamma_{d}(A \cap B) \geq \gamma_{d}(A) \gamma_{d}(B)
$$

where $\gamma_{d}$ is the standard Gaussian measure on $\mathbb{R}^{d}$. The case $d=1$ is trivial, since $A$ and $B$ are centered intervals, hence one is contained in the other. The case $d=2$ was proved by Pitt [7]. For higher dimensional cases, there are only partial results. For instance, in [8] it was shown that (1.1) holds if $A$ and $B$ are ellipsoids, which was soon generalized by Hargé [3] to allow one of them to be an arbitrary symmetric convex set. Hargé's proof relies on the modified Ornstein-Uhlenbeck semigroup and the properties of log-concave functions. A rather short proof of Hargé's result was presented in [2], by making use of the deep results in the theory of optimal transport, that is, the optimal transport map, which pushes forward the Gaussian measure $\gamma_{d}$ to a probability measure $\nu$ having a log-concave density with respect to $\gamma_{d}$, is a contraction (see [1]). In [4], the author obtained a correlation inequality for the Gaussian measure via a formula for Itô-Wiener chaos expansion. Li W.V. [5] presented a weaker form of the correlation inequality (1.1), which is useful to show the existence of small ball constants. For a more detailed survey of the studies on (1.1), see [6, Section 2.4].

In this note we consider two special cases of the correlation inequality. It is clear that we only need to consider bounded subsets of $\mathbb{R}^{d}$. In the sequel, we always assume that the sets are bounded and closed. First we prove the following result.

\footnotetext{
*Email: luodj@amss.ac.cn
} 
Theorem 1.1. Let $\mathrm{d} \mu=\rho(|x|) \mathrm{d} x$ be a probability measure on $\mathbb{R}^{d}$ with $\rho \in C\left(\mathbb{R}_{+},(0, \infty)\right)$. Suppose $A \subset \mathbb{R}^{d}$ is convex and the origin $0 \in A$. Then for any ball $B$ centered at the origin, we have

$$
\mu(A \cap B) \geq \mu(A) \mu(B) .
$$

Clearly the Gaussian measure $\gamma_{d}$ is a special case of $\mu$ considered above. The new point here is that the set $A$ does not have to be symmetric, at the price of the regularity on $B$. Theorem 1.1 is a slight generalization of [2, Proposition 1]; the latter requires that the origin 0 is the unique fixed point of all the isometries which leave $A$ (globally) invariant. Remark also that our proof (see Section 2) uses purely elementary analysis, while the one in [2] relies on the result in the theory of optimal transport.

If we want to prove the correlation inequality for more general sets $B$ other than the balls (e.g. the ellipsoids), then some additional conditions have to be imposed on the set $A$.

Theorem 1.2. Assume that $\mu$ is a product probability measure: $\mu=\prod_{i=1}^{d} \mu_{i}$, where $\mathrm{d} \mu_{i}=$ $\rho_{i}\left(\left|x_{i}\right|\right) \mathrm{d} x_{i}$ with $\rho_{i} \in C\left(\mathbb{R}_{+},(0, \infty)\right)$. Let $A \subset \mathbb{R}^{d}$ be a convex set with the following property: $x \in A$ implies that its projections on all the coordinate hyperplanes also belong to $A$. Then for any ellipsoid

$$
B=\left\{x \in \mathbb{R}^{d}: \frac{x_{1}^{2}}{a_{1}^{2}}+\cdots+\frac{x_{d}^{2}}{a_{d}^{2}} \leq 1\right\},
$$

where $a_{1}, \cdots, a_{d}$ are positive constants, we have $\mu(A \cap B) \geq \mu(A) \mu(B)$.

This result will be proved in Section 3. It is easy to see that the set $A$ considered in Theorem 1.2 contains the origin 0 . An example for the set $A$ is $\left\{x=\left(x_{1}, \cdots, x_{d}\right) \in \mathbb{R}^{d}: \forall i=\right.$ $1, \cdots, d, x_{i} \geq 0$ and $\left.\sum_{i=1}^{d} x_{i} \leq 1\right\}$. We would like to mention that Theorem 1.2 still holds for more general set $B$, see Remark 3.3.

A nonnegative function $f: \mathbb{R}^{d} \rightarrow \mathbb{R}_{+}$is called log-concave if for any $x, y \in \mathbb{R}^{d}$ and $0<\lambda<1$, $f(\lambda x+(1-\lambda) y) \geq f(x)^{\lambda} f(y)^{1-\lambda}$. For any convex set $A \subset \mathbb{R}^{d}$, one easily concludes that the indicator function $\mathbf{1}_{A}$ is log-concave. The Gaussian correlation inequality (1.1) has the following functional version: for any log-concave and symmetric functions $f, g$, it holds

$$
\gamma_{d}(f g) \geq \gamma_{d}(f) \gamma_{d}(g)
$$

Here $\gamma_{d}(f)=\int_{\mathbb{R}^{d}} f \mathrm{~d} \gamma_{d}$. Following the method of [2, Section 3], we show in the last section that (1.2) holds if $f$ is log-concave and $g=\varphi(\langle\Sigma x, x\rangle)$, where $\varphi \in C\left(\mathbb{R}_{+}, \mathbb{R}_{+}\right)$is a decreasing function and $\Sigma$ is a positive definite matrix. So we give an alternative proof to [3, Theorem 2]. By an approximation argument, we obtain again Hargé's result.

\section{Proof of Theorem 1.1}

In this section we prove Theorem 1.1. In fact we will prove a more general result. To this end, we introduce a class of functions on $\mathbb{R}^{d}$ :

$$
\mathcal{C}_{d}=\left\{f \in C_{c}\left(\mathbb{R}^{d}, \mathbb{R}_{+}\right): \forall c>0,\{f>c\} \text { is convex and } \forall x \in \mathbb{R}^{d}, f(x) \leq f(0)\right\} .
$$

Let $S^{d-1}$ be the unit sphere in $\mathbb{R}^{d}$. For a bounded measurable function $g$ on $\mathbb{R}^{d}$, define $\mu(g)=$ $\int_{\mathbb{R}^{d}} g \mathrm{~d} \mu$. We have the following simple observations.

Lemma 2.1. Let $f \in \mathcal{C}_{d}$ and $f \neq 0$. Then

(i) for any $\theta \in S^{d-1}$, the function $t \mapsto f(t \theta)$ is decreasing on $\mathbb{R}_{+}$; 
(ii) $f(0)>\mu(f)$.

Proof. (i) Suppose that there are $t_{1}<t_{2}$ such that $f\left(t_{1} \theta\right)<f\left(t_{2} \theta\right)$. Consider the set $E:=$ $\left\{f>\left[f\left(t_{1} \theta\right)+f\left(t_{2} \theta\right)\right] / 2\right\}$. Then $0, t_{2} \theta \in E$ but $t_{1} \theta \in E^{c}$, which contradicts the fact that $E$ is convex.

(ii) By the definition of the class $\mathcal{C}_{d}$,

$$
f(0)-\mu(f)=\int_{\mathbb{R}^{d}}(f(0)-f(x)) \mathrm{d} \mu(x) \geq 0 .
$$

If $f(0)=\mu(f)$, then $f(x) \equiv f(0)$ for all $x \in \mathbb{R}^{d}$, which is impossible. Hence $f(0)>\mu(f)$.

Now we prove

Theorem 2.2. Assume that $\mathrm{d} \mu=\rho(|x|) \mathrm{d} x$ is a probability measure on $\mathbb{R}^{d}$ with $\rho \in C\left(\mathbb{R}_{+},(0, \infty)\right)$. For any $f \in \mathcal{C}_{d}$ and any ball $B$ centered at the origin, it holds

$$
\mu\left(f \mathbf{1}_{B}\right) \geq \mu(f) \mu(B) .
$$

Proof. Obviously we can assume $\mu(f)>0$. For $t \geq 0$, let $B_{t}$ be the ball centered at the origin with radius $t$. Define the function

$$
\Phi(t)=\mu\left(f \mathbf{1}_{B_{t}}\right)-\mu(f) \mu\left(B_{t}\right), \quad t \geq 0 .
$$

First we show that $\Phi$ is positive when $t$ is sufficiently small and large. By Lemma 2.1(ii), there is $t_{0}>0$ such that for all $x \in B_{t_{0}}, f(x)>\mu(f)$. Thus for any $t \in\left(0, t_{0}\right)$,

$$
\Phi(t)=\int_{B_{t}}[f(x)-\mu(f)] \mathrm{d} \mu(x)>0 .
$$

When $t$ is big enough such that $\operatorname{supp}(f) \subset B_{t}$, we have

$$
\Phi(t)=\mu(f)-\mu(f) \mu\left(B_{t}\right)=\mu(f) \mu\left(B_{t}^{c}\right)>0 .
$$

Next we compute the derivative $\Phi^{\prime}(t)$. We have for $h>0$,

$$
\mu\left(f \mathbf{1}_{B_{t+h}}\right)-\mu\left(f \mathbf{1}_{B_{t}}\right)=\int_{B_{t+h} \backslash B_{t}} f(x) \mathrm{d} \mu(x)=\int_{B_{t+h} \backslash B_{t}} f(x) \rho(|x|) \mathrm{d} x .
$$

Using the spherical coordinate, the above equality can be written as

$$
\begin{aligned}
\mu\left(f \mathbf{1}_{B_{t+h}}\right)-\mu\left(f \mathbf{1}_{B_{t}}\right) & =\int_{t}^{t+h}\left(\int_{S^{d-1}} f(r \theta) \rho(|r \theta|) \mathrm{d} \sigma(\theta)\right) r^{d-1} \mathrm{~d} r \\
& =\int_{t}^{t+h}\left(\int_{S^{d-1}} f(r \theta) \mathrm{d} \sigma(\theta)\right) \rho(r) r^{d-1} \mathrm{~d} r,
\end{aligned}
$$

where $\sigma$ is the volume measure on $S^{d-1}$. Since the functions $f$ and $\rho$ are continuous, dividing both sides by $h$ and letting $h \rightarrow 0$ lead to

$$
\frac{\mathrm{d}}{\mathrm{d} t} \mu\left(f \mathbf{1}_{B_{t}}\right)=\rho(t) t^{d-1} \int_{S^{d-1}} f(t \theta) \mathrm{d} \sigma(\theta) .
$$

Similarly we have $\frac{\mathrm{d}}{\mathrm{d} t} \mu\left(B_{t}\right)=\rho(t) t^{d-1} \sigma\left(S^{d-1}\right)$. Therefore

$$
\begin{aligned}
\Phi^{\prime}(t) & =\rho(t) t^{d-1} \int_{S^{d-1}} f(t \theta) \mathrm{d} \sigma(\theta)-\mu(f) \rho(t) t^{d-1} \sigma\left(S^{d-1}\right) \\
& =\rho(t) t^{d-1} \int_{S^{d-1}}[f(t \theta)-\mu(f)] \mathrm{d} \sigma(\theta) .
\end{aligned}
$$


From this expression, it is clear that $\Phi^{\prime}$ is continuous. For $t>0$ small enough, we conclude from (2.2) and Lemma 2.1(ii) that $\Phi^{\prime}(t)>0$. Let $t_{1}=\inf \left\{t>0: \Phi^{\prime}(t)=0\right\}$. Then $\int_{S^{d-1}}\left[f\left(t_{1} \theta\right)-\right.$ $\mu(f)] \mathrm{d} \sigma(\theta)=0$. By Lemma 2.1(i), for any $t>t_{1}$,

$$
\int_{S^{d-1}}[f(t \theta)-\mu(f)] \mathrm{d} \sigma(\theta) \leq \int_{S^{d-1}}\left[f\left(t_{1} \theta\right)-\mu(f)\right] \mathrm{d} \sigma(\theta)=0 .
$$

Hence $\Phi^{\prime}(t) \leq 0$. It follows that $\Phi(t)$ is increasing on $\left[0, t_{1}\right]$ and decreasing on $\left[t_{1}, \infty\right)$. Combining this with the fact that $\Phi(t)>0$ when $t$ is sufficiently small and large, we complete the proof.

Now we are ready to prove Theorem 1.1.

Proof of Theorem 1.1. We will construct a sequence of functions, belonging to $\mathcal{C}_{d}$, which converge to $\mathbf{1}_{A}$. Let $\operatorname{dist}(\cdot, A)$ be the distance function to $A$. For $n \geq 1$, define

$$
f_{n}(x)=1-n\left[n^{-1} \wedge \operatorname{dist}(x, A)\right], \quad x \in \mathbb{R}^{d} .
$$

Then it is clear that $f_{n} \in C_{c}\left(\mathbb{R}^{d}, \mathbb{R}_{+}\right), 0 \leq f_{n} \leq 1$ and the restriction $\left.f_{n}\right|_{A} \equiv 1$. Since $0 \in A$, we have for all $x \in \mathbb{R}^{d}, f_{n}(x) \leq 1=f_{n}(0)$. It remains to show that for any $c \in[0,1),\left\{f_{n}>c\right\}$ is convex. In fact,

$$
\left\{f_{n}>c\right\}=\left\{x \in \mathbb{R}^{d}: \operatorname{dist}(x, A)<(1-c) / n\right\} .
$$

If $x, y \in\left\{f_{n}>c\right\}$, then $\operatorname{dist}(x, A) \vee \operatorname{dist}(y, A)<(1-c) / n$. Thus there are $x_{0}, y_{0} \in A$ such that $\left|x-x_{0}\right| \vee\left|y-y_{0}\right|<(1-c) / n$. For any $\lambda \in(0,1)$, we have by the convexity of $A, \lambda x_{0}+(1-\lambda) y_{0} \in A$. Moreover,

$$
\left|(\lambda x+(1-\lambda) y)-\left(\lambda x_{0}+(1-\lambda) y_{0}\right)\right| \leq \lambda\left|x-x_{0}\right|+(1-\lambda)\left|y-y_{0}\right|<(1-c) / n .
$$

Therefore $\operatorname{dist}(\lambda x+(1-\lambda) y, A)<(1-c) / n$; equivalently, $\lambda x+(1-\lambda) y \in\left\{f_{n}>c\right\}$. This means that $\left\{f_{n}>c\right\}$ is convex.

Now applying Theorem 2.2 to $f_{n}$, we have

$$
\mu\left(f_{n} \mathbf{1}_{B}\right) \geq \mu\left(f_{n}\right) \mu(B), \quad \text { for all } n \geq 1 .
$$

Since $f_{n} \downarrow \mathbf{1}_{A}$ on $\mathbb{R}^{d}$, by the monotone convergence theorem, letting $n \rightarrow \infty$ completes the proof.

\section{Proof of Theorem 1.2}

In order to prove Theorem 1.2, we introduce another family of functions:

$$
\begin{gathered}
\overline{\mathcal{C}}_{d}=\left\{f \in C_{c}\left(\mathbb{R}^{d}, \mathbb{R}_{+}\right):\right. \\
: i \in\{1, \cdots, d\} \text { and }\left(x_{1}, \cdots, x_{i-1}, x_{i+1}, \cdots, x_{d}\right) \in \mathbb{R}^{d-1} \text { fixed, } \\
\text { the function } \left.x_{i} \mapsto f\left(x_{1}, \cdots, x_{i-1}, x_{i}, x_{i+1}, \cdots, x_{d}\right) \in \mathcal{C}_{1}\right\},
\end{gathered}
$$

where $\mathcal{C}_{1}$ is the class of functions defined in (2.1) for $d=1$. We have

Theorem 3.1. Assume that $\mu$ is a product probability measure: $\mu=\prod_{i=1}^{d} \mu_{i}$, where $\mathrm{d} \mu_{i}=$ $\rho_{i}\left(\left|x_{i}\right|\right) \mathrm{d} x_{i}$ with $\rho_{i} \in C\left(\mathbb{R}_{+},(0, \infty)\right)$. Let $B$ be an ellipsoid:

$$
B=\left\{x \in \mathbb{R}^{d}: \frac{x_{1}^{2}}{a_{1}^{2}}+\cdots+\frac{x_{d}^{2}}{a_{d}^{2}} \leq 1\right\},
$$

where $a_{1}, \cdots, a_{d}$ are positive constants. Then for any $f \in \overline{\mathcal{C}}_{d}$, the following inequality holds:

$$
\mu\left(f \mathbf{1}_{B}\right) \geq \mu(f) \mu(B) .
$$


Proof. We will prove this result by induction on the dimension $d$. When $d=1$, this theorem is a special case of Theorem 1.1. Now suppose that the assertion is true in the $d-1$ dimensional case. Denote by $\mu_{(d-1)}=\prod_{i=1}^{d-1} \mu_{i}$ the product measure on $\mathbb{R}^{d-1}$. By Fubini's theorem,

$$
\begin{aligned}
\mu\left(f \mathbf{1}_{B}\right) & =\int_{B} f \mathrm{~d}\left(\mu_{(d-1)} \times \mu_{d}\right) \\
& =\int_{-a_{d}}^{a_{d}} \mathrm{~d} \mu_{d}\left(x_{d}\right) \int_{B_{d-1}\left(x_{d}\right)} f\left(x_{1}, \cdots, x_{d-1}, x_{d}\right) \mathrm{d} \mu_{(d-1)}\left(x_{1}, \cdots, x_{d-1}\right),
\end{aligned}
$$

where $B_{d-1}\left(x_{d}\right)$ is a $d-1$ dimensional ellipsoid:

$$
B_{d-1}\left(x_{d}\right)=\left\{\left(x_{1}, \cdots, x_{d-1}\right) \in \mathbb{R}^{d-1}: \frac{x_{1}^{2}}{a_{1}^{2}}+\cdots+\frac{x_{d-1}^{2}}{a_{d-1}^{2}} \leq 1-\frac{x_{d}^{2}}{a_{d}^{2}}\right\} .
$$

Notice that for fixed $x_{d} \in\left[-a_{d}, a_{d}\right], f\left(\cdot, x_{d}\right) \in \overline{\mathcal{C}}_{d-1}$. Using the induction hypothesis, we have

$$
\int_{B_{d-1}\left(x_{d}\right)} f\left(x_{1}, \cdots, x_{d-1}, x_{d}\right) \mathrm{d} \mu_{(d-1)}\left(x_{1}, \cdots, x_{d-1}\right) \geq \mu_{(d-1)}\left(f\left(\cdot, x_{d}\right)\right) \mu_{(d-1)}\left(B_{d-1}\left(x_{d}\right)\right) .
$$

Therefore by (3.1),

$$
\mu\left(f \mathbf{1}_{B}\right) \geq \int_{-a_{d}}^{a_{d}} \mu_{(d-1)}\left(f\left(\cdot, x_{d}\right)\right) \mu_{(d-1)}\left(B_{d-1}\left(x_{d}\right)\right) \mathrm{d} \mu_{d}\left(x_{d}\right) .
$$

The function $\left[-a_{d}, a_{d}\right] \ni x_{d} \mapsto \mu_{(d-1)}\left(B_{d-1}\left(x_{d}\right)\right)$ is even and $\mu_{(d-1)}\left(B_{d-1}\left(a_{d}\right)\right)=0$. We extend it to a function on $\mathbb{R}$ by setting $\mu_{(d-1)}\left(B_{d-1}\left(x_{d}\right)\right) \equiv 0$ for $\left|x_{d}\right|>a_{d}$. Then the above inequality becomes

$$
\begin{aligned}
\mu\left(f \mathbf{1}_{B}\right) & \geq \int_{-\infty}^{\infty} \mu_{(d-1)}\left(f\left(\cdot, x_{d}\right)\right) \mu_{(d-1)}\left(B_{d-1}\left(x_{d}\right)\right) \mathrm{d} \mu_{d}\left(x_{d}\right) \\
& =\left(\int_{-\infty}^{0}+\int_{0}^{\infty}\right) \mu_{(d-1)}\left(f\left(\cdot, x_{d}\right)\right) \mu_{(d-1)}\left(B_{d-1}\left(x_{d}\right)\right) \mathrm{d} \mu_{d}\left(x_{d}\right) .
\end{aligned}
$$

We denote by $I_{1}$ and $I_{2}$ the two integrals on the right hand side of (3.2). Note that the even function $x_{d} \mapsto \mu_{(d-1)}\left(B_{d-1}\left(x_{d}\right)\right)$ is decreasing on $\mathbb{R}_{+}$. On the other hand, for any fixed $\left(x_{1}, \cdots, x_{d-1}\right) \in \mathbb{R}^{d-1}$, by the definition of the class $\overline{\mathcal{C}}_{d}$ and Lemma 2.1(i), the function $x_{d} \mapsto$ $f\left(x_{1}, \cdots, x_{d-1}, x_{d}\right)$ is decreasing (resp. increasing) on $\mathbb{R}_{+}$(resp. $\left.\mathbb{R}_{-}=(-\infty, 0]\right)$. Hence the same is true for $x_{d} \mapsto \mu_{(d-1)}\left(f\left(\cdot, x_{d}\right)\right)$. Applying the FKG inequality (see Lemma 3.2 below) to $2 \mu_{d}$ on $(-\infty, 0]$ leads to

$$
\begin{aligned}
I_{1} & \geq \frac{1}{2}\left(2 \int_{-\infty}^{0} \mu_{(d-1)}\left(f\left(\cdot, x_{d}\right)\right) \mathrm{d} \mu_{d}\left(x_{d}\right)\right)\left(2 \int_{-\infty}^{0} \mu_{(d-1)}\left(B_{d-1}\left(x_{d}\right)\right) \mathrm{d} \mu_{d}\left(x_{d}\right)\right) \\
& =\left(\int_{-\infty}^{0} \mu_{(d-1)}\left(f\left(\cdot, x_{d}\right)\right) \mathrm{d} \mu_{d}\left(x_{d}\right)\right)\left(\int_{-\infty}^{\infty} \mu_{(d-1)}\left(B_{d-1}\left(x_{d}\right)\right) \mathrm{d} \mu_{d}\left(x_{d}\right)\right),
\end{aligned}
$$

where the equality follows from the symmetry of the integrand the the measure $\mu_{d}$. Similarly we have

$$
I_{2} \geq\left(\int_{0}^{\infty} \mu_{(d-1)}\left(f\left(\cdot, x_{d}\right)\right) \mathrm{d} \mu_{d}\left(x_{d}\right)\right)\left(\int_{-\infty}^{\infty} \mu_{(d-1)}\left(B_{d-1}\left(x_{d}\right)\right) \mathrm{d} \mu_{d}\left(x_{d}\right)\right) .
$$

Combining this with (3.2) and (3.3), we conclude that

$$
\begin{aligned}
\mu\left(f \mathbf{1}_{B}\right) & \geq\left(\int_{-\infty}^{\infty} \mu_{(d-1)}\left(f\left(\cdot, x_{d}\right)\right) \mathrm{d} \mu_{d}\left(x_{d}\right)\right)\left(\int_{-\infty}^{\infty} \mu_{(d-1)}\left(B_{d-1}\left(x_{d}\right)\right) \mathrm{d} \mu_{d}\left(x_{d}\right)\right) \\
& =\mu(f) \mu(B) .
\end{aligned}
$$

Therefore the result holds as well in the $d$ dimensional case. The proof is complete. 
Lemma 3.2 (FKG inequality). Let $-\infty \leq a, b \leq \infty$ and $\nu$ be a probability measure on $[a, b]$. Assume $f$ and $g$ are two bounded increasing (or decreasing) functions on $[a, b]$, then

$$
\int_{a}^{b} f g \mathrm{~d} \nu \geq\left(\int_{a}^{b} f \mathrm{~d} \nu\right)\left(\int_{a}^{b} g \mathrm{~d} \nu\right)
$$

Proof. For any $x, y \in[a, b]$, since both $f$ and $g$ are increasing (or decreasing) functions on $[a, b]$, we have

$$
(f(x)-f(y))(g(x)-g(y)) \geq 0 .
$$

As the two functions are bounded, we can integrate the above inequality on $[a, b]^{2}$ with respect to $\nu \times \nu$ and obtain

$$
\int_{[a, b]^{2}}(f(x)-f(y))(g(x)-g(y)) \mathrm{d}(\nu \times \nu)(x, y) \geq 0 .
$$

Expanding the product gives the desired result.

Remark 3.3. The proof of Theorem 3.1 works for more general set $B$. Indeed, for $i=1, \cdots, d$, let $f_{i} \in C\left(\mathbb{R}_{+}, \mathbb{R}_{+}\right)$be a strictly increasing function such that $f_{i}(0)=0$. Then the result of Theorem 3.1 still holds for the set

$$
B=\left\{x \in \mathbb{R}^{d}: f_{1}\left(\left|x_{1}\right|\right)+\cdots+f_{d}\left(\left|x_{d}\right|\right) \leq 1\right\} .
$$

Notice that $B$ can even be non-convex. For example, when $d=2$ and $f_{1}(t)=f_{2}(t)=\sqrt{t}$ for $t \geq 0$, then $B=\left\{x \in \mathbb{R}^{2}: \sqrt{\left|x_{1}\right|}+\sqrt{\left|x_{2}\right|} \leq 1\right\}$ is clearly not convex.

Now we are in the position to prove Theorem 1.2. We focus on the case $d \geq 2$ (the case $d=1$ has been proved in Theorem 1.1).

Proof of Theorem 1.2. Consider the approximations $f_{n}$ of the indicator function $\mathbf{1}_{A}$ defined in (2.3). In order to apply Theorem 3.1, we have to show that for every $n \geq 1, f_{n} \in \overline{\mathcal{C}}_{d}$. For simplicity of notations, we assume $i=1$, that is, for any $x^{\prime}=\left(x_{2}, \cdots, x_{d}\right) \in \mathbb{R}^{\bar{d}-1}$ fixed, we need to prove that the function $x_{1} \mapsto f_{n}\left(x_{1}, x^{\prime}\right) \in \mathcal{C}_{1}$, where $\mathcal{C}_{1}$ is defined in (2.1). For any $c>0$,

$$
I:=\left\{x_{1} \in \mathbb{R}: f_{n}\left(x_{1}, x^{\prime}\right)>c\right\}=\left\{x_{1} \in \mathbb{R}: \operatorname{dist}\left(\left(x_{1}, x^{\prime}\right), A\right)<(1-c) / n\right\} .
$$

If $x_{1}, \bar{x}_{1} \in I, x_{1}<\bar{x}_{1}$, then $\operatorname{dist}\left(\left(x_{1}, x^{\prime}\right), A\right) \vee \operatorname{dist}\left(\left(\bar{x}_{1}, x^{\prime}\right), A\right)<(1-c) / n$. Since $A$ is convex, as in the proof of Theorem 1.1, we can show that

$$
\operatorname{dist}\left(\lambda\left(x_{1}, x^{\prime}\right)+(1-\lambda)\left(\bar{x}_{1}, x^{\prime}\right), A\right)<(1-c) / n
$$

for all $\lambda \in(0,1)$. That is, $\operatorname{dist}\left(\left(\lambda x_{1}+(1-\lambda) \bar{x}_{1}, x^{\prime}\right), A\right)<(1-c) / n$. Consequently, for all $\lambda \in(0,1), \lambda x_{1}+(1-\lambda) \bar{x}_{1} \in I$. This means that $I$ is an interval, hence it is convex.

Next we prove that $0 \in I$ whenever $I$ is nonempty. Indeed, if $x_{1} \in I$, then $\operatorname{dist}\left(\left(x_{1}, x^{\prime}\right), A\right)<$ $(1-c) / n$. Hence there is $y=\left(y_{1}, y^{\prime}\right) \in A$ such that $\left|\left(x_{1}, x^{\prime}\right)-\left(y_{1}, y^{\prime}\right)\right|<(1-c) / n$. By the property of $A$, we have $\left(0, y^{\prime}\right) \in A$. Moreover

$$
\left|\left(0, x^{\prime}\right)-\left(0, y^{\prime}\right)\right| \leq\left|\left(x_{1}, x^{\prime}\right)-\left(y_{1}, y^{\prime}\right)\right|<(1-c) / n .
$$

Therefore $\operatorname{dist}\left(\left(0, x^{\prime}\right), A\right)<(1-c) / n$, that is, $0 \in I$. Now if there is $x_{1} \in \mathbb{R}$ such that $f_{n}\left(x_{1}, x^{\prime}\right)>$ $f_{n}\left(0, x^{\prime}\right)$, then consider the interval

$$
\tilde{I}=\left\{f_{n}\left(\cdot, x^{\prime}\right)>\left(f_{n}\left(x_{1}, x^{\prime}\right)+f_{n}\left(0, x^{\prime}\right)\right) / 2\right\} .
$$

We have $x_{1} \in \tilde{I}$ but $0 \in \tilde{I}^{c}$, which is a contradiction with the result that we have just proved. Hence $f_{n}\left(0, x^{\prime}\right) \geq f_{n}\left(x_{1}, x^{\prime}\right)$ for all $x_{1} \in \mathbb{R}$. Therefore the function $x_{1} \mapsto f_{n}\left(x_{1}, x^{\prime}\right) \in \mathcal{C}_{1}$. Summing up these arguments, we conclude that $f_{n} \in \overline{\mathcal{C}}_{d}$.

Now applying Theorem 3.1 to $f_{n}$, we obtain $\mu\left(f_{n} \mathbf{1}_{B}\right) \geq \mu\left(f_{n}\right) \mu(B)$ for all $n \geq 1$. Letting $n \rightarrow \infty$ gives the desired result. 


\section{$4 \quad$ A special case of $(1.2)$}

In the present section, we follow the method in [2] (see p.265) and prove that the inequality (1.2) holds if $g$ is the composition of a decreasing function and a positive definite quadratic form. This gives an alternative proof to [3, Theorem 2].

Theorem 4.1. Assume that $f \in C\left(\mathbb{R}^{d}, \mathbb{R}_{+}\right)$is a log-concave and symmetric function. Let $\Sigma$ be a positive definite matrix and $\varphi \in C\left(\mathbb{R}_{+}, \mathbb{R}_{+}\right)$a decreasing function. Then

$$
\int_{\mathbb{R}^{d}} f(x) \varphi(\langle\Sigma x, x\rangle) \mathrm{d} \gamma_{d}(x) \geq\left(\int_{\mathbb{R}^{d}} f(x) \mathrm{d} \gamma_{d}(x)\right)\left(\int_{\mathbb{R}^{d}} \varphi(\langle\Sigma x, x\rangle) \mathrm{d} \gamma_{d}(x)\right) .
$$

Proof. Consider the Gaussian probability measure

$$
\mathrm{d} \mu=\frac{1}{(2 \pi)^{n / 2} \sqrt{\operatorname{det}(\Sigma)}} e^{-\left\langle\Sigma^{-1} x, x\right\rangle / 2} \mathrm{~d} x,
$$

where $\operatorname{det}(\Sigma)$ is the determinant of $\Sigma$. Then (4.1) is equivalent to

$$
\int_{\mathbb{R}^{d}} f\left(\sqrt{\Sigma^{-1}} x\right) \varphi\left(|x|^{2}\right) \mathrm{d} \mu(x) \geq\left(\int_{\mathbb{R}^{d}} f\left(\sqrt{\Sigma^{-1}} x\right) \mathrm{d} \mu(x)\right)\left(\int_{\mathbb{R}^{d}} \varphi\left(|x|^{2}\right) \mathrm{d} \mu(x)\right) .
$$

Since $f$ is log-concave and symmetric, it is easy to see that $f(x) \leq f(0)$ for all $x \in \mathbb{R}^{d}$, hence $C_{f}:=\int_{\mathbb{R}^{d}} f\left(\sqrt{\Sigma^{-1}} x\right) \mathrm{d} \mu(x)<+\infty$. We introduce the probability measure $\mu_{f}$ defined by

$$
\mathrm{d} \mu_{f}=\frac{1}{C_{f}} f\left(\sqrt{\Sigma^{-1}} x\right) \mathrm{d} \mu(x) .
$$

Hence it is sufficient to prove that

$$
\int_{\mathbb{R}^{d}} \varphi\left(|x|^{2}\right) \mathrm{d} \mu_{f}(x) \geq \int_{\mathbb{R}^{d}} \varphi\left(|x|^{2}\right) \mathrm{d} \mu(x) .
$$

Now let $T$ be the optimal transport map pushing forward the Gaussian measure $\mu$ to $\mu_{f}$, i.e. $\mu_{f}=\mu \circ T^{-1}$. Since the density function $\frac{1}{C_{f}} f\left(\sqrt{\Sigma^{-1}} x\right)$ is also log-concave, we deduce from Caffarelli's result (see [1]) that $T$ is a contraction. Moreover by the symmetry of the density function, we have $T(-x)=-T(x)$; particularly, $T(0)=0$. Therefore, $|T(x)| \leq|x|$ for all $x \in \mathbb{R}^{d}$. As a result,

$$
\begin{aligned}
\int_{\mathbb{R}^{d}} \varphi\left(|x|^{2}\right) \mathrm{d} \mu_{f}(x) & =\int_{\mathbb{R}^{d}} \varphi\left(|x|^{2}\right) \mathrm{d}\left(\mu \circ T^{-1}\right)(x) \\
& =\int_{\mathbb{R}^{d}} \varphi\left(|T(x)|^{2}\right) \mathrm{d} \mu(x) \geq \int_{\mathbb{R}^{d}} \varphi\left(|x|^{2}\right) \mathrm{d} \mu(x),
\end{aligned}
$$

where the last inequality follows from the fact that $\varphi$ is a decreasing function. (4.3) is proved.

If we take $\varphi(t)=e^{-t / 2}$ for $t \geq 0$, then Theorem 4.1 reduces to [8, Proposition 2] (see p.352). Moreover by approximating the indicator function of a symmetric convex set, we can reprove Hargé's result [3].

Corollary 4.2. Let $A \subset \mathbb{R}^{d}$ be any symmetric convex set and $B$ be the ellipsoid $\left\{x \in \mathbb{R}^{d}\right.$ : $\langle\Sigma x, x\rangle \leq 1\}$. Then

$$
\gamma_{d}(A \cap B) \geq \gamma_{d}(A) \gamma_{d}(B)
$$


Proof. We consider again the sequence of approximating functions $f_{n}$ defined in (2.3). First we show that $f_{n}$ is $\log$-concave for every $n \geq 1$. Since the set $A$ is convex, it is easy to see that the distance function $\operatorname{dist}(\cdot, A)$ is also convex. Hence for any $x, y \in \mathbb{R}^{d}$ and $0<\lambda<1$,

$$
\operatorname{dist}(\lambda x+(1-\lambda) y, A) \leq \lambda \operatorname{dist}(x, A)+(1-\lambda) \operatorname{dist}(y, A) .
$$

In order to show that $f_{n}(\lambda x+(1-\lambda) y) \geq f_{n}(x)^{\lambda} f_{n}(y)^{1-\lambda}$, it is enough to consider the case $f_{n}(x) \wedge f_{n}(y)>0$, that is, $\operatorname{dist}(x, A) \vee \operatorname{dist}(y, A)<1 / n$. Therefore by (4.4),

$$
1-n \operatorname{dist}(\lambda x+(1-\lambda) y, A) \geq \lambda[1-n \operatorname{dist}(x, A)]+(1-\lambda)[1-n \operatorname{dist}(y, A)] .
$$

In other words,

$$
f_{n}(\lambda x+(1-\lambda) y) \geq \lambda f_{n}(x)+(1-\lambda) f_{n}(y) .
$$

Now using Young's inequality (for any $a, b \geq 0$ and $p, q>1$ such that $p^{-1}+q^{-1}=1$, it holds $\left.a b \leq \frac{a^{p}}{p}+\frac{b^{q}}{q}\right)$, we obtain

$$
\lambda f_{n}(x)+(1-\lambda) f_{n}(y) \geq f_{n}(x)^{\lambda} f_{n}(y)^{1-\lambda} .
$$

Combining this with (4.5), we obtain the log-concavity of $f_{n}$.

The symmetry of the set $A$ implies that $f_{n}$ is also symmetric. Now applying Theorem 4.1 to the functions $f_{n}$ and letting $n \rightarrow \infty$, we arrive at

$$
\int_{\mathbb{R}^{d}} \mathbf{1}_{A}(x) \varphi(\langle\Sigma x, x\rangle) \mathrm{d} \gamma_{d}(x) \geq \gamma_{d}(A)\left(\int_{\mathbb{R}^{d}} \varphi(\langle\Sigma x, x\rangle) \mathrm{d} \gamma_{d}(x)\right) .
$$

Next define

$$
\varphi_{n}(t)= \begin{cases}1, & t \in[0,1] \\ 1-n(t-1), & 1<t<1+n^{-1} \\ 0, & t \geq 1+n^{-1}\end{cases}
$$

Then $\varphi_{n}(t) \downarrow \mathbf{1}_{[0,1]}(t)$ as $n \rightarrow \infty$. Replacing $\varphi$ by $\varphi_{n}$ in (4.6) and letting $n \rightarrow \infty$, we finally get the desired result.

\section{References}

[1] Caffarelli L. Monotonicity properties of optimal transportation and the FKG and related inequalities. Comm. Math. Phys. 214 (2000), 547-563.

[2] Cordero-Erausquin Dario. Some applications of mass transport to Gaussian-type inequalities. Arch. Rational Mech. Anal. 161 (2002), 257-269.

[3] Hargé Gilles. A particular case of correlation inequality for the Gaussian measure. Ann. Probab. 27 (1999), 1939-1951.

[4] $\mathrm{Hu}$ Yaozhong. Ito-Wiener chaos expansion with exact residual and correlation, variance inequalities. J. Theoret. Probab. 10 (1997), 835-848.

[5] Li Wenbo V. A Gaussian correlation inequality and its applications to small ball probabilities. Elect. Comm. in Probab. 4 (1999), 111-118.

[6] Li Wenbo V., Shao Qi-Man. Gaussian Processes: Inequalities, Small Ball Probabilities and Applications. STOCHASTIC PROCESSES: THEORY AND METHODS. Handbook of Statistics, Vol. 19, 533-598, Elsevier, New York (2001). 
[7] Pitt L.D. A Gaussian correlation inequality for symmetric convex sets. Ann. Probab. 5 (1977), 470-474.

[8] Schechtman G., Schlumprecht T. and Zinn J. On the Gaussian measure of intersection of symmetric convex sets. Ann. Probab. 26 (1998), 346-357. 